\title{
Die fotografische Inszenierung des Verbrechens. Ein Album aus Auschwitz / Das Höcker-Album. Auschwitz durch die Linse der SS
}

\section{Valentine Devulder}

\section{(2) OpenEdition}

\section{Journals}

Édition électronique

URL : http://journals.openedition.org/ifha/10862

DOI : $10.4000 /$ ifha. 10862

ISSN : 2198-8943

Éditeur

IFRA - Institut franco-allemand (sciences historiques et sociales)

Référence électronique

Valentine Devulder, «Die fotografische Inszenierung des Verbrechens. Ein Album aus Auschwitz / Das Höcker-Album. Auschwitz durch die Linse der SS », Revue de l'IFHA [En ligne], Date de recension, mis en ligne le 18 août 2020, consulté le 24 septembre 2020. URL : http://journals.openedition.org/ifha/ 10862 ; DOI : https://doi.org/10.4000/ifha.10862

Ce document a été généré automatiquement le 24 septembre 2020.

(C)IFHA 


\title{
Die fotografische Inszenierung des Verbrechens. Ein Album aus Auschwitz / Das Höcker-Album. Auschwitz durch die Linse der SS
}

\author{
Valentine Devulder
}

\section{RÉFÉRENCE}

Tal Bruttmann, Stefan Hördler, Christoph Kreutzmüller, Die fotografische Inszenierung des Verbrechens. Ein Album aus Auschwitz, Darmstadt : Wbg Academic Verlag, 2019, $305 p$., $60 €$ Christophe Busch, Stefan Hördler, Robert Jan van Pelt (dir.), Das Höcker-Album. Auschwitz durch die Linse der SS, Darmstadt : Wbg Academic Verlag, 2020, 340p., $25 €$

Bien que chacun des deux ouvrages se suffise à lui-même en terme d'apport historiographique et d'intérêt historique, il était particulièrement intéressant de les lire de front tant leurs objets d'études se complètent.

L'ouvrage de Busch, Hördler et van Pelt porte sur l'album connu sous le nom d'« Album Auschwitz » ou de « Lili Jacob », du nom de la rescapée juive hongroise qui le trouve en 1945, qui contient 188 photographies capturant les derniers instants des Juifs et Juives de Hongrie déportés en mai-juin 1944. Cet album est rendu public en 1983 par L. Jacob elle-même, qui en fait don à Yad Vashem. L'ouvrage de Bruttmann, Hördler et Kreutzmüller s'intéresse quant à lui à un album contemporain du premier mais découvert bien plus tard, celui désormais dénommé « Album Höcker ». Du nom de l'officier SS Karl Höcker auquel appartenait l'objet, il est composé de 116 photographies en noir et blanc, représentant plusieurs groupes d'officiers et d'auxiliaires SS pendant leur temps libre, notamment au lieu de villégiature SS de la « Solahütte », à l'été 1944. Il fut rendu public en 2007 quand son détenteur, un officier américain anonyme, en fait don au United States Holocaust Memorial Museum. 
L'ouvrage portant sur « l'Album de Lili Jacob » s'intéresse d'abord au contexte des photographies, qui portent toutes sur les premiers jours de déportation des Juifs et Juives de Hongrie à Auschwitz en mai 1944. Les auteurs se penchent ensuite sur les trois personnages principaux qui gravitent autour de l'album : Ernst Hoffmann et Bernhard Walter, les deux officiers SS à l'origine des photographies, et Lili Jacob, qui trouve l'album en 1945 et y ajoute deux clichés, représentant les chambres à gaz du camp. Viennent ensuite les photos elles-mêmes, mais reproduites trois fois dans l'ouvrage. Une première fois, sans commentaire, reprenant l'ordre, la pagination, les titres et commentaires de l'album d'origine. À noter que l'ordre d'origine est thématique : l'arrivée des convois, la sélection pour le camp de travail ou vers la chambre à gaz, la désinfection des survivant à la sélection, l'entrée dans le camp de travail et le triage des effets des nouveaux détenus. Les photos sont une nouvelle fois reproduites, dans une volonté de déconstruire à la fois l'ordre et l'intention des photographes. Les auteurs de l'ouvrage soulignent la présence d'une violence constante, même si elle n'est jamais physiquement représentée, car le fait même de photographier des hommes et des femmes dans un moment si terrible est un acte de violence. À cela s'ajoute le fait que les photographes capturent les derniers instants de milliers d'hommes et de femmes, en pleine connaissance de l'assassinat qui les attend, quand eux ne le savent pas. Les photographies sont reproduites une troisième fois, cette fois dans un effort de reconstruction chronologique, thématique et spatial, et avec la volonté d'identifier les hommes et les femmes photographiés, en suivant les sélections et les arrivées de convoi, certains datables (notamment celui de Lili Jacob elle-même et de ses proches), d'autres non. La dernière partie de l'ouvrage, consacrée à la « reconstruction » de l'album évite relativement bien l'écueil spéculatif auquel on pourrait être confronté, et ce malgré quelques hypothèses (cette femme portait deux manteaux, était-elle malade ? ce petit garçon porte un manteau bien trop grand pour lui, lui a-t-on donné car il avait froid ou était trempé ?).

La lecture parallèle de l'ouvrage de Busch, Hördler et van Pelt offre une complémentarité d'autant plus forte que les deux ouvrages partagent un auteur, Stefan Hördler. Cela permet à chaque livre d'explorer un peu l'autre album et d'en souligner les parallèles historiques.

L'ouvrage consacré à l'album de Höcker est un ouvrage collectif composé d'articles, ce qui explique sa division thématique, chaque auteur et autrice se penchant sur un aspect particulier de l'étude de l'album de Höcker; il ne s'agit pas d'une étude systématique de déconstruction photographie par photographie.

Dans l'ensemble, l'ouvrage revient sur la vie et le personnage de Karl Höcker en détail, identifié comme le propriétaire et le créateur de l'album (mais pas l'auteur des photos, probablement plusieurs personnes, qui ne sont jamais identifiées). Bien qu'il ait été un officier SS important dans la hiérarchie national-socialiste, il est véritablement redécouvert avec l'étude de son album. Second du commandant Martin Weiß des camps de concentration de Neuengamme, Arbeitsdorf puis Majdanek entre 1940 et mai 1944, date à laquelle il est transféré à Auschwitz, il devient le second du commandant Richard Baer jusqu'en 1945. Les photos qu'il prend datent presque toutes de l'été 1944 et représentent uniquement des officiers SS ou des auxiliaires féminines de la SS, quand l'album de Lili Jacob se concentre sur les populations déportées (la présence des officiers SS est fréquente mais n'est pas le sujet principal). Elles sont principalement prises à la "Solahütte », un lieu de villégiature pour les officiers SS et leurs familles, qui se trouve à une trentaine de kilomètres au sud de Auschwitz et a été construit par des 
détenus du camp dès l'automne 1940, dans les environs des villages de MiĘdzybrodzkie et de PorĄbka. Pendant toute la durée de la guerre, de petits groupes de détenus et détenues sont forcés de travailler à l'entretien de la Solahütte. Pourtant, aucun des détenus n'apparaît jamais sur aucune des photos de Höcker.

Ces photos sont très déconcertantes, car elles remettent en question l'image démoniaque habituelle des nazis : Höcker se photographie en discutant et en riant avec Richard Baer, avec Joseph Mengele ou encore Eno Lolling pour ne citer que quelques figures connues, en jouant avec son chien, en mangeant des myrtilles avec de jeunes dactylographes auxiliaires SS, ou encore allongé sur des transatlantiques.

Les auteurs et autrices posent des questions intéressantes : comment le monde représenté dans l'album de Höcker peut-il être le même que celui de l'album de Lili Jacob ? Comment est-il possible de concilier de telles vies parallèles, si opposées mais tellement entremêlées ? Comment se met en place la dissonance cognitive nécessaire aux nazis pour « faire leur travail»?

Au-delà de l'apport visuel, l'album de Höcker apporte de nouvelles informations précieuses : par exemple, les papiers de l'officier SS Anton Thumann n'indiquaient jamais qu'il avait servi à Auschwitz, ce que démentent les photographies de Höcker. Elles permettent également de comparer les personnages officiers SS jusque là non encore identifiés sur les photos de l'album de Lili Jacob et de les identifier, notamment quand leur présence sur la « Rampe » les incrimine directement du meurtre des Juifs hongrois. Ce sont aussi les seules photos connues de Joseph Mengele en uniforme SS dans le camp de Auschwitz et en compagnie de ses camarades.

$\mathrm{Au}$ fil de ses articles, l'ouvrage interroge également la place des femmes au sein de l'appareil nazi concentrationnaire. S. Cushman les divise en quatre catégories : les gardiennes, les fonctionnaires administratives, les infirmières et doctoresses, et les auxiliaires de la Kommandantur. On voit à plusieurs reprises ces femmes dans les photos prises par Höcker, principalement de très jeunes dactylographes d'une vingtaine d'années. S. Cushman insiste sur leur rôle important dans la mise en place du système concentrationnaire : si elles ne prirent pas part aux actes de violence et aux assassinats de masse, leur responsabilité n'en est pas moins fortement engagée, notamment par leur engagement volontaire et motivé comme auxiliaires SS.

Chacun des deux ouvrages est très documenté et se suffit largement à lui-même. Cependant, la lecture parallèle et la connaissance simultanée des deux albums de photographies permettent d'appréhender pleinement l'ambiguïté des photographies de Höcker. L'absence des détenus du camp d'Auschwitz est criante quand on se souvient que la déportation des 450000 Juifs et Juives de Hongrie se déroule à la même période et est orchestrée par les mêmes hommes et femmes qui sont représentés dans l'album de Höcker. Le premier album, qui documente les populations déportées, est un acte de violence en lui-même, quand le second album se veut un recueil de souvenirs d'une période de joyeuse camaraderie. Il est alors difficile de se rendre compte que les visages épanouis du second album sont les tortionnaires des hommes et femmes du premier album. 
INDEX

Index chronologique : Époque contemporaine

Thèmes : Histoire sociale, Histoire militaire, Histoire de l'art

\section{AUTEURS}

VALENTINE DEVULDER

EHESS 